\title{
Necessary Conditions for the Existence of Utility Maximizing Strategies under Transaction Costs
}

\author{
Paolo Guasoni \\ Walter Schachermayer \\ Boston University and University of Pisa \\ Vienna University of Technology
}

February 3, 2004

\begin{abstract}
For any utility function with asymptotic elasticity equal to one, we construct a market model in countable discrete time, such that the utility maximization problem with proportional transaction costs admits no solution.

This proves that the necessity of the reasonable asymptotic elasticity condition, established by Kramkov and Schachermayer KS99] in the frictionless case, remains valid also in the presence of transaction costs.
\end{abstract}

\section{Introduction}

An economic agent endowed with initial capital $x$ trades in a financial market so as to maximize the expected utility of his terminal wealth at a fixed time horizon $T$. In general, we can write this optimization problem as:

$$
\max _{H} E\left[U\left(x+V_{T}(H)\right)\right]
$$

where $V_{T}(H)$ represents the portfolio value obtained with the trading rule $H$, which varies in a suitable set of "admissible" strategies, defined so as to exclude arbitrage through doubling schemes. In most cases, and also in the present paper, this is achieved through the solvability constraint $x+V_{t}(H) \geq 0$ a.s. for all $t \in[0, T]$, a natural choice for utility functions defined on positive values, such as $U(X)=\log x$ and $U(x)=x^{\alpha} / \alpha$, with $\alpha \in(-\infty, 1) \backslash\{0\}$.

We adopt the definitions from [KS99], to which we refer for unexplained notation. We assume that the utility function $U: \mathbb{R}_{+} \mapsto \mathbb{R}$ satisfies the Inada conditions:

Assumption 1.1. A utility function $U: \mathbb{R}_{+} \mapsto \mathbb{R}$ satisfies the regularity (Inada) conditions if it is strictly increasing, strictly concave, continuously differentiable, $U^{\prime}(0)=\infty$ and $U^{\prime}(\infty)=0$. 
In this setting it was shown in KS99] that a necessary and sufficient condition on the utility function $U$ for the existence of an optimal solution in any financial market model is the condition that the asymptotic elasticity of $U$ is strictly less than 1 :

$$
A E_{+\infty}(U)=\limsup _{x \rightarrow \infty} \frac{x U^{\prime}(x)}{U(x)}<1
$$

When this condition fails, one can always construct a market model leading to a utility maximization problem which admits no solution (provided the initial capital $x$ is strictly greater than a given critical value $x^{*}$ (see [KS99, section 5)).

This phenomenon can be explained by looking at maximizing strategies: as observed in [KS99, when (1) fails and $x>x^{*}$, the economic agent can approach the maximal expected utility as follows: he/she splits the initial capital $x$ into two parts. An amount equal to the critical value $x^{*}$ is used to set up the strategy which is optimal for $x=x^{*}$. The remainder $x-x^{*}$ is used to buy a very risky bet, which will most likely return nothing, although with a tiny chance it will win a huge sum.

The idea is that there is a sequence $B_{n}$ of such bets, becoming more and more risky as $n \rightarrow \infty$, yielding a strictly increasing (as $n \rightarrow \infty$ ) contribution to the expected utility appearing in $(\mathrm{P})$, which tends to a finite limit. The agent would therefore like to pass to the limit of $\left(B_{n}\right)_{n=1}^{\infty}$, but this turns out to be impossible, as in the limit this would be a bet which yields with probability 0 a gain $\infty$, which does not make sense. This is, very roughly speaking, the idea underlying Proposition 5.2 in [KS99] (compare also Example 3.2 in Sch02]).

The aim of the present paper is to investigate the problem of existence of the optimizer in $(\mathrm{P})$ in the presence of transaction costs: we assume that there is one risky asset, modeled by the $\mathbb{R}$-valued price process $\left(S_{t}\right)_{t \in[0, T]}$ and a bond, normalized to $B_{t}=1$ such that, whenever we buy or sell the risky asset, transaction costs of $k$ times the value of the trade are due, where $0 \leq k<1$ is given. We remark that with the methods below we could also treat the case when transaction costs are different for buying and selling: but, mainly for the sake of notational convenience, we assume that they agree.

We investigate, to which extent the results obtained in [KS99] for the frictionless case $k=0$ carry over to the case $k>0$. On the positive side, in was shown in Gua02 that, also in the presence of transaction costs, condition (1) is sufficient for the existence of the optimizer in $(\mathrm{P})$. The question addressed in the present paper is whether under transaction costs condition (1) is still necessary, as in the frictionless case, or whether the risky bets described above are wiped out by transaction costs.

We show that the nonexistence phenomenon persists: for any utility function $U$ such that:

$$
\limsup _{x \rightarrow \infty} \frac{x U^{\prime}(x)}{U(x)}=1
$$


and any $x^{*}>0$, we construct a market model where the utility maximization problem $(\mathrm{P})$ does not admit a solution for initial capital $x>x^{*}$. In fact, we shall construct a complete market, defined on a countable probability space and indexed by countable discrete time displaying this phenomenon.

The main result of this paper therefore reads as follows:

Proposition 1.2. Let $U$ satisfy Assumption 1.1 and (2), and let $0 \leq k<1$. Denoting by $t_{n}=1-\frac{1}{n+1}$, there exists a stochastic process $\left(X_{t_{n}}\right)_{n=0}^{\infty}$, based on the filtered probability space $\left(\Omega, \mathcal{F},\left(\mathcal{F}_{n}\right)_{n=0}^{\infty}, P\right)$, where $\mathcal{F}_{n}=\sigma\left(\left(X_{t_{i}}\right)_{i=0}^{n}\right)$ and $\mathcal{F}=\mathcal{F}_{\infty}=\sigma\left(\vee_{n=0}^{\infty} \mathcal{F}_{n}\right)$, such that:

i) $u(x)=\sup \left\{E\left[U\left(x+V_{1}^{k}(H)\right)\right]: H\right.$ admissible $\}$ is finite for all $x>0$.

ii) Problem $(\mathrm{P})$ has a solution if and only if $x \leq x^{*}$

iii) $u^{\prime}(x)$ is constant for $x \geq x^{*}$.

The construction is done in two steps: in section 2 we construct a frictionless model with one risky asset $S$ in countable discrete time, which serves the same purpose without transaction costs. Unlike the counterexample given in [KS99, based on the dual problem, here the model is constructed explicitly for the primal problem (compare also Example 3.2 in [Sch02]). The construction described below implies the existence of maximizing sequences with positive holdings in the risky asset, which will be crucial for our following analysis.

In section 3 we modify the construction to find a financial market, now denoted by $\left(X_{t_{n}}\right)_{n=0}^{\infty}$, which does the same purpose in the presence of transaction costs $k>0$. The idea is that $X_{t_{n}}-X_{t_{n-1}}$ is a suitable modification of $(-1)^{n}\left(S_{t_{n}}-S_{t_{n-1}}\right)$. This will imply that, in order to find a "good" trading strategy, one should be long in the risky asset at even and short at odd times. Of course, such a behavior generates a lot of transaction costs, but the "suitable modification" is done precisely in such a way to set off these transaction costs. In this way we succeed in carrying over the arguments from the frictionless case to that of a market with transaction costs.

\section{The Frictionless Example}

We recall some notation from [KS99]. Fix an $\mathbb{R}$-valued semimartingale $\left(S_{t}\right)_{t \in[0, T]}$, where $T>0$. We denote by

$$
\mathcal{A}_{x}=\left\{H \text { predictable, } S \text {-integrable }: x+\int_{0}^{t} H_{s} d S_{s} \geq 0 \quad \text { a.s. for all } 0 \leq t \leq T\right\}
$$

the set of admissible strategies with initial capital $x$, and by

$$
\mathcal{X}(x)=\left\{X: X \leq x+\int_{0}^{T} H_{t} d S_{t}: H \in \mathcal{A}_{x}\right\}
$$


the corresponding set of superreplicable claims. The value function, or indirect utility, of $(\mathrm{P})$ is then:

$$
u(x)=\sup _{X \in \mathcal{X}(x)} E[U(X)]
$$

In the present paper, we embed the discrete time model $\left(\left(X_{t_{n}}\right)_{n=0}^{\infty}, \Omega, \mathcal{F},\left(\mathcal{F}_{n}\right)_{n=0}^{\infty}, P\right)$ in the continuous time interval $[0, T]$ (with $T=1$ ) through the usual right-continuous extension $X_{t}=X_{t_{n}}$ for $t \in\left[t_{n}, t_{n+1}\right)$.

In this section we give a proof for the following variant of Example 5.2 in [KS99]:

Proposition 2.1. Let $U$ satisfy Assumption 1.1, and (2). For any $x^{*}>0$, there is a complete financial market $\left(S_{t_{n}}\right)_{n=0}^{\infty}$, based on a countable filtered probability space $\left(\Omega, \mathcal{F},\left(\mathcal{F}_{n}\right)_{n=0}^{\infty}, P\right)$ such that:

i) $u(x)<\infty$, for all $x>0$,

ii) For $x>0$, problem $(\mathrm{P})$ admits an optimizer $\hat{X}(x)=x+(\hat{H}(x) \cdot S)_{T}$, with $\hat{H}(x) \in \mathcal{A}_{x}$, if and only if $x \leq x^{*}$,

iii) $u^{\prime}(x)$ is constant for $x \geq x^{*}$.

As indicated in the introduction, the idea of the construction is that for $x>x^{*}$ the optimal investment will roughly be $x^{*}$ to finance $\hat{X}\left(x^{*}\right)$ and the remaining funds $x-x^{*}$ to buy a bet which with high probability yields 0 , while with low probability $p_{n}$ it yields a gain $\left(x-x^{*}\right) \xi_{n}^{n}$ such that $p_{n} U\left(\left(x-x^{*}\right) \xi_{n}^{n}\right) \sim y\left(x-x^{*}\right)$, where $y=u^{\prime}\left(x^{*}\right)$. To achieve this, it will be convenient to introduce some notation:

Definition 2.2. A financial market $\left(\Omega, \mathcal{F},\left(\mathcal{F}_{n}\right)_{n=0}^{\infty}, P,\left(S_{t_{n}}\right)_{n=0}^{\infty}\right)$ is a simple jump model if:

i) $\Omega=\left(\cup_{n=1}^{\infty} A_{n}\right) \cup B$, where $\left(A_{n}\right)_{n=1}^{\infty}$ and $B$ are disjoint nonempty sets such that $P\left(A_{n}\right)=$ $p_{n}$ for some numbers $p_{n}>0$, where $\sum_{n=1}^{\infty} p_{n}=1-P(B)<1$.

ii) $\mathcal{F}_{n}=\sigma\left(\left(A_{k}\right)_{k=1}^{n}\right)$, for $n \geq 0$.

iii) The risky asset process $S_{t_{n}}$ is defined by:

$$
S_{t_{n}}=\sum_{k=1}^{n} \alpha_{k}\left(1_{A_{k}}-q_{k}\right) 1_{\Omega \backslash\left(\cup_{i=1}^{k-1} A_{i}\right)}
$$

where the numbers $\left(\alpha_{n}\right)_{n=1}^{\infty}$ and $\left(q_{n}\right)_{n=1}^{\infty}$ satisfy $\sum_{n=1}^{\infty} \alpha_{n}<\infty, q_{n}>0$ for all $n$, and $\sum_{n=1}^{\infty} q_{n}<1$.

Remark 2.3. It is immediate from the above definition that a simple jump model is complete, and the unique equivalent martingale measure $Q$ for $S$ is given by $Q\left(A_{n}\right)=q_{n}$ and $Q(B)=$ $1-\sum_{n=1}^{\infty} q_{n}$. 
We break the proof of Proposition 2.1 into two Lemmata. In the first one, we show that the quantities $\left(p_{n}\right)_{n=1}^{\infty}$ and $\left(q_{n}\right)_{n=1}^{\infty}$ in Definition 2.2 can be chosen so that the optimal terminal payoff on $S^{N}$ with initial capital $x^{* *}$ prescribes an investment of $x^{* *}-x_{N}$ in the Arrow-Debreu asset $1_{A_{N}}$. As $N$ increases to infinity, the value functions $u^{N}\left(x^{* *}\right)$ increase to $u\left(x^{* *}\right)$, also finite-valued, and $y^{N}$, the Lagrange multipliers associated to the above problems, increase to a finite value $y^{\infty}$. Finally, the actual optimizing strategies $H^{N}$ always require positive holdings in the asset $S$.

¿From now on, we shall write, for notational convenience, $S_{n}$ (respectively $H_{n}, X_{n}$ ) in place of $S_{t_{n}}\left(\operatorname{resp} . H_{t_{n}}, X_{t_{n}}\right)$.

Lemma 2.4. Let $U$ be a utility function satisfying Assumption 1.1 and (2). Let $x^{*}>0$, $x^{* *}>x^{*}$, and let $\left(x_{n}\right)_{n=1}^{\infty}$, with $x_{1}>0$, be a sequence strictly increasing to $x^{*}$. Consider a simple jump model, as in Definition 2.2, and denote by $\left(S_{n}^{N}\right)_{n=1}^{\infty}$ the process $\left(S_{n}\right)_{n=1}^{\infty}$ stopped at $N$, and by $u^{N}(x)$ and $u(x)$ the value functions of the utility maximization problems on $S$ and $S^{N}$ respectively. Finally, denote by $\hat{X}_{T}^{N}\left(x^{* *}\right)=\xi_{1}^{N} 1_{A_{1}}+\cdots+\xi_{N}^{N} 1_{A_{N}}+\mu^{N} 1_{B_{N}}$ the optimal terminal payoff on $S^{N}$ with initial capital $x^{* *}$.

We can choose the quantities $\left(p_{n}\right)_{n=1}^{\infty}$ and $\left(q_{n}\right)_{n=1}^{\infty}$ such that:

i) $q_{N} \xi_{N}^{N}=x^{* *}-x_{N}$

ii) $0<q_{n}<\frac{p_{n}}{2}$ and $\sum_{n=1}^{\infty} p_{n} \leq \frac{1}{2}$.

iii) $u(x)=\lim _{N \rightarrow \infty} u^{N}(x)<\infty$ for all $x>0$, and $y^{\infty}=\lim _{N \rightarrow \infty} y^{N}<\infty$, where $y^{N}=\left(u^{N}\right)^{\prime}\left(x^{* *}\right)$ and $y^{\infty}=u^{\prime}\left(x^{* *}\right)$.

iv) For all $x>0$ and $N \geq 1$, the optimal strategies $\left(\hat{H}_{n}^{N}\right)_{n=1}^{N}$, defined by:

$$
\hat{X}_{T}^{N}(x)=x+\sum_{n=1}^{N} \hat{H}_{n}^{N}\left(S_{n}-S_{n-1}\right)
$$

satisfy the condition $\hat{H}_{n}^{N} \geq 0$ a.s. for all $n \leq N$.

Proof. Note that without loss of generality we may assume $U(\infty)>0$, as adding a constant to $U$ does not change the optimization problem.

First we need to introduce some notation. Let us fix strictly positive numbers $\left(\varepsilon_{n}\right)_{n=1}^{\infty}$, such that $\prod_{n=1}^{\infty}\left(1+\varepsilon_{n}\right)<2$, and denote by $I=\left(U^{\prime}\right)^{-1}$. Denote also the set

$$
\Xi=\left\{x: U(x)>0, \frac{x U^{\prime}(x)}{U(x)}>\frac{1}{2}\right\}
$$

which is open by Assumption 1.1 and unbounded by assumption (2).

We shall construct our model so that the following properties hold: 
(a) $\sum_{n=1}^{N} p_{n}<\frac{1}{2}-2^{-(N+1)}$

(b) $q_{n}<\frac{p_{n}}{2}$ for $1 \leq n \leq N$

(c) $\xi_{N}^{N} q_{N}=x^{* *}-x_{N}$

(d) $U^{\prime}\left(\frac{x_{N+1}-x_{N}}{2\left(x^{* *}-x_{N}\right)} \xi_{N}^{N}\right)<U^{\prime}\left(\xi_{N}^{N}\right)\left(1+\varepsilon_{N}\right)$

(e) $I\left(y \frac{q_{N}}{p_{N}}\right) \in \Xi$ for all $y \in\left[y^{N}, y^{\infty}\right]$

We proceed by induction on $N$. Consider first $N=1$ : we shall find $p_{1}$ and $q_{1}$, which determine $\xi_{1}^{1}, \mu^{1}$ and $y^{1}$ so that

$$
\begin{aligned}
\hat{X}^{1}\left(x^{* *}\right) & =\xi_{1}^{1} 1_{A_{1}}+\mu^{1} 1_{B_{1}} \quad \text { and } \\
y^{1} & =\left(u^{1}\right)^{\prime}\left(x^{* *}\right)
\end{aligned}
$$

For fixed parameter $\xi_{1}^{1} \in \Xi$, we obtain the first order conditions for the optimal portfolio satisfying $(c)$ for the one-period problem $S^{1}$ by solving the following equations for the unknowns $p_{1}, q_{1}, y^{1}, \mu^{1}$, for given parameter $\xi_{1}^{1}$ :

$$
\begin{aligned}
\xi_{1}^{1} q_{1} & =x^{* *}-x_{1} \\
\xi_{1}^{1} q_{1}+\mu^{1}\left(1-q_{1}\right) & =x^{* *} \\
U^{\prime}\left(\xi_{1}^{1}\right) & =y^{1} \frac{q_{1}}{p_{1}} \\
U^{\prime}\left(\mu^{1}\right) & =y^{1} \frac{1-q_{1}}{1-p_{1}}
\end{aligned}
$$

Let us check that this system has indeed a solution: $q_{1}$ is determined by (4), and $\mu^{1}$ by (5). (6) determines $p_{1}$ in terms of $y^{1}$, and plugging in (7) we obtain:

$$
y^{1}=\frac{U^{\prime}\left(\mu^{1}\right)}{1+\frac{x^{* *}-x_{1}}{\xi_{1}^{1}}\left(\frac{1}{U^{\prime}\left(\xi^{1}\right)}-1\right)}
$$

Recalling that $\lim _{x \rightarrow \infty} \frac{U(x)}{x}=0$ and that:

$$
\frac{1}{2} \leq \liminf _{\substack{x \rightarrow \infty \\ x \in \Xi}} \frac{x U^{\prime}(x)}{U(x)} \leq \limsup _{\substack{x \rightarrow \infty \\ x \in \Xi}} \frac{x U^{\prime}(x)}{U(x)} \leq 1
$$

we obtain that:

$$
\lim _{\substack{\xi_{1}^{1} \rightarrow \infty \\ \xi_{1}^{1} \in \Xi}} y^{1}\left(\xi_{1}^{1}\right)=\lim _{\substack{\xi_{1}^{1} \rightarrow \infty \\ \xi_{1}^{1} \in \Xi}} \frac{U^{\prime}\left(\mu^{1}\right) U\left(\xi_{1}^{1}\right)}{U\left(\xi_{1}^{1}\right)+\left(x^{* *}-x_{1}\right)\left(\frac{U\left(\xi_{1}^{1}\right)}{\xi_{1}^{1} U^{\prime}\left(\xi_{1}^{1}\right)}-\frac{U\left(\xi_{1}^{1}\right)}{\xi_{1}^{1}}\right)}=\eta<\infty
$$


¿From (6) and $(9)$ it follows that:

$$
p_{1}=y^{1} \frac{x^{* *}-x_{1}}{U^{\prime}\left(\xi_{1}^{1}\right) \xi_{1}^{1}}<2 y^{1} \frac{x^{* *}-x_{1}}{U\left(\xi_{1}^{1}\right)}
$$

and therefore we can assume that $p_{1}<\frac{1}{4}$ and $q_{1}<\frac{p_{1}}{2}$ (by (6)) for $\xi_{1}^{1}$ large enough.

Finally, we apply Lemma A.1 to $\varepsilon=\min \left(\varepsilon_{1}, \frac{x_{2}-x_{1}}{2\left(x^{* *}-x_{1}\right)}\right)$, which provides some large $\xi_{1}^{1}$ for which (d) is satisfied, and condition (e) is obtained up to a change to smaller $\left(\varepsilon_{n}\right)_{n=2}^{\infty}$.

It is worthwhile to resume the present procedure. For the parameter $\xi_{1}^{1}$ we obtain from the necessary first-order conditions (4)-(7) how the quantities $\xi_{1}^{1}, p_{1}, q_{1}, y^{1}$ and $\mu^{1}$ have to be related. Then we turn around and specify (for a large value of $\xi_{1}^{1}$ ) the parameters $p_{1}, q_{1}$ in our model as we have just obtained them. It then follows that for this model $\xi_{1}^{1}$ and $\mu^{1}$ indeed define the optimal portfolio, while $y^{1}$ is the associated Lagrange multiplier satisfying $\left(u^{1}\right)^{\prime}\left(x^{* *}\right)=y^{1}$.

Let us now pass to the induction step. Suppose that we have constructed $\left(p_{i}\right)_{i=1}^{N}$ and $\left(q_{i}\right)_{i=1}^{N}$ such that the resulting quantities $\left(\xi_{i}^{N}\right)_{i=1}^{N}$ and $y^{N}=\left(u^{N}\right)^{\prime}\left(x^{* *}\right)$ satisfy (a)-(e) above. Let us construct $p_{N+1}, q_{N+1}$, which in turn determine $\mu^{N+1}, y^{N+1}$ and $\left(\xi_{i}^{N+1}\right)_{i=1}^{N+1}$ such that the same assumptions are satisfied for $N+1$.

Again, we leave $\xi=\xi_{N+1}^{N+1}$ as a free parameter to be fixed later, and solve the $N+4$ equations:

$$
\begin{aligned}
\xi_{N+1}^{N+1} q_{N+1} & =x^{* *}-x_{N+1} \\
\sum_{n=1}^{N} \xi_{n}^{N+1} q_{n}+\xi_{N+1}^{N+1} q_{N+1}+\mu^{N+1}\left(1-\sum_{n=1}^{N+1} q_{n}\right) & =x^{* *} \\
U^{\prime}\left(\xi_{n}^{N+1}\right) & =y^{N+1} \frac{q_{n}}{p_{n}} \quad \text { for } n=1 \ldots N+1 \\
U^{\prime}\left(\mu^{N+1}\right) & =y^{N+1} \frac{1-\sum_{n=1}^{N+1} q_{n}}{1-\sum_{n=1}^{N+1} p_{n}}
\end{aligned}
$$

in the unknowns $q_{N+1}, p_{N+1}, y^{N+1}, \mu^{N+1}$ and $\left(\xi_{i}^{N+1}\right)_{i=1}^{N}$. Again, $q_{N+1}$ is determined by (10). If we fix $y^{N+1}=y$ we are left with $N+2$ equations (12) and (13)), each one involving exactly one of the $N+2$ unknowns $\left(\xi_{i}^{N+1}\right)_{i=1}^{N}, p_{N+1}$ and $\mu^{N+1}$, which admit a unique solution depending on $\xi$ and $y$. We write $\xi_{n}^{N+1}(y)$ and $\mu^{N+1}(y)$ to indicate their dependence on $y$. Now, denote the quantity on the left side of (11),

$$
G(y)=\sum_{n=1}^{N} \xi_{n}^{N+1}(y) q_{n}+\left(x^{* *}-x_{N+1}\right)+\mu^{N+1}(y)\left(1-\sum_{n=1}^{N+1} q_{n}\right)
$$

Observe that $G$ is a strictly decreasing function for $y \geq y^{N}$. In fact, from $(12)-(13)$ we have that $\xi_{n}^{N+1}=I\left(y \frac{q_{n}}{p_{n}}\right)$, and since $I$ is a decreasing function, the first summation in (14) is 
decreasing. To prove the claim, it remains to show that $\mu^{N+1}(y)$ is also decreasing. Solving (12) for $p_{N+1}$, we have that:

$$
p_{N+1}=y \frac{x^{* *}-x_{N+1}}{\xi U^{\prime}(\xi)}
$$

and substituting in $(13)$, we obtain:

$$
\mu^{N+1}(y)=I\left(y \frac{1-\sum_{n=1}^{N} q_{n}-\frac{x^{* *}-x_{N+1}}{\xi}}{1-\sum_{n=1}^{N} p_{n}-y \frac{x^{* *}-x_{N+1}}{\xi U^{\prime}(\xi)}}\right)=I\left(\frac{a y}{b-c y}\right)
$$

where $a=1-\sum_{n=1}^{N} q_{n}-\frac{x^{* *}-x_{N+1}}{\xi}, b=1-\sum_{n=1}^{N} p_{n}$, and $c=\frac{x^{* *}-x_{N+1}}{\xi U^{\prime}(\xi)}$ are all positive numbers. Since $\frac{a y}{b-c y}$ is an increasing function, the claim follows.

For $y=y^{N}$ we have that $\xi_{n}^{N+1}\left(y^{N}\right)=\xi_{n}^{N}$ for $n=1, \ldots, N$, and $\mu^{N+1}\left(y^{N}\right)$ and $\mu^{N}$ are arbitrarily close for large $\xi$. In fact, by the inductive hypothesis:

$$
\sum_{n=1}^{N} \xi_{n}^{N} q_{n}+\mu^{N}\left(1-\sum_{n=1}^{N} q_{n}\right)=x^{* *}
$$

we obtain that:

$$
G\left(y^{N}\right)=x^{* *}+\left(x^{* *}-x_{N+1}\right)+\mu^{N+1}\left(1-\sum_{n=1}^{N+1} q_{n}\right)-\mu^{N}\left(1-\sum_{n=1}^{N} q_{n}\right)
$$

For $\xi$ large enough, $\mu^{N+1}$ approaches to $\mu^{N}$ and $q_{N+1}$ approaches to zero, therefore we can assume that $G\left(y^{N}\right)>x^{* *}$. For $y=y^{N}\left(1+\varepsilon_{n}\right)$, since $\xi_{n}^{N+1}(y)$ is decreasing, we have that:

$$
\xi_{n}^{N+1}\left(y^{N}\left(1+\varepsilon_{N}\right)\right) \leq \xi_{n}^{N+1}\left(y^{N}\right)=\xi_{n}^{N}\left(y^{N}\right) \text { for } n=1 \ldots N-1
$$

Furthermore, by the inductive hypothesis (d):

$$
y^{N}\left(1+\varepsilon_{N}\right)=U^{\prime}\left(\xi_{N}^{N}\right)\left(1+\varepsilon_{N}\right)>U^{\prime}\left(\frac{x_{N+1}-x_{N}}{2\left(x^{* *}-x_{N}\right)} \xi_{N}^{N}\right)
$$

and hence:

$$
\xi_{N}^{N+1}\left(y\left(1+\varepsilon_{N}\right)\right)<\frac{x_{N+1}-x_{N}}{2\left(x^{* *}-x_{N}\right)} \xi_{N}^{N}
$$


Substituting (15) and (16) in (14), we obtain:

$$
\begin{aligned}
& G\left(y^{N}\left(1+\varepsilon_{N}\right)\right)< \\
&< \sum_{n=1}^{N-1} \xi_{n}^{N} q_{n}+x^{* *}+\frac{x_{N+1}-x_{N}}{2\left(x^{* *}-x_{N}\right)} \xi_{N}^{N} q_{N}+\left(x^{* *}-x_{N+1}\right)+\mu^{N+1}\left(y^{N}\left(1+\varepsilon_{N}\right)\right)\left(1-\sum_{n=1}^{N+1} q_{n}\right)= \\
&=-\left(x^{* *}-x_{N}\right)+\frac{x_{N+1}-x_{N}}{2}+\left(x^{* *}-x_{N+1}\right)+\mu^{N+1}\left(1-\sum_{n=1}^{N+1} q_{n}\right)-\mu^{N}\left(1-\sum_{n=1}^{N} q_{n}\right)= \\
&=-\frac{1}{2}\left(x_{N+1}-x_{N}\right)+\varphi(\xi)
\end{aligned}
$$

where

$$
\varphi(\xi)=\mu^{N+1}(\xi)\left(1-\sum_{n=1}^{N} q_{n}-q_{N+1}(\xi)\right)-\mu^{N}\left(1-\sum_{n=1}^{N} q_{n}\right)
$$

is infinitesimal, in the sense that $\lim _{\xi \rightarrow \infty} \varphi(\xi)=0$.

Therefore, for a sufficiently large $\xi, G\left(y^{N}\left(1+\varepsilon_{N}\right)\right)<x^{* *}$, and there is a unique value $y^{N+1} \in\left(y^{N}, y^{N}\left(1+\varepsilon^{N}\right)\right)$ such that $G(y)=x^{* *}$. Hence, using this value of $y^{N+1}$ in equations (10 11 12 13), we obtain a solution to the entire system, and in particular we obtain the desired values for $p_{N+1}$ and $q_{N+1}$. To check (b), (d) and (e), we apply Lemma A.1 to $\varepsilon=\min \left(\varepsilon_{N}, \frac{x_{N+2}-x_{N+1}}{2\left(x^{* *}-x_{N+1}\right)}\right)$, and obtain some large $\xi$ such that:

$$
U^{\prime}\left(\frac{x_{N+2}-x_{N+1}}{2\left(x^{* *}-x_{N+1}\right)} \xi\right)<U^{\prime}(\xi)\left(1+\varepsilon_{N+1}\right)
$$

which implies (d), and up a change to smaller $\left(\varepsilon_{n}\right)_{n=N+1}^{\infty}$ we obtain (e). Finally, we let $\xi$ be large enough so that $q_{N+1}<\frac{p_{N+1}}{2}$, and the induction hypotheses are satisfied. This completes the construction.

The sequence $\left(y^{N}\right)_{N=1}^{\infty}$ increases to $y^{\infty}<\infty$. In fact, the assumption $\prod_{n=1}^{\infty}\left(1+\varepsilon_{n}\right)<2$ guarantees that:

$$
y^{N} \leq y^{1} \prod_{n=2}^{N}\left(1+\varepsilon_{n}\right) \leq 2 y^{1}
$$

Then by 12 it follows that $\left(\xi_{n}^{N}\right)_{N=n}^{\infty}$ decreases to the value $\xi_{n}$ satisfying $U^{\prime}\left(\xi_{n}\right)=y^{\infty} \frac{q_{n}}{p_{n}}$. Similarly, we can prove that $\mu^{N}$ decreases to the value $\mu$ which satisfies $U^{\prime}(\mu)=y^{\infty} r_{n}$, where $r_{n}=\frac{1-\sum_{k=1}^{n} q_{k}}{1-\sum_{k=1}^{n} p_{k}}$. In fact, by 13 it is sufficient to prove that $r_{n}$ is increasing. To see this, we use the assumption $q_{n}<\frac{p_{n}}{2}$, which implies:

$$
r_{N+1}=\frac{1-\sum_{n=1}^{N} q_{n}-q_{N+1}}{1-\sum_{n=1}^{N} p_{n}-p_{N+1}}>\frac{1-\sum_{n=1}^{N} q_{n}-\frac{p_{N+1}}{2}}{1-\sum_{n=1}^{N} p_{n}-p_{N+1}}>\frac{1-\sum_{n=1}^{N} q_{n}}{1-\sum_{n=1}^{N} p_{n}}=r_{N}
$$


where the last inequality is equivalent to $1-\sum_{n=1}^{N} q_{n} \geq \frac{1-\sum_{n=1}^{N} p_{n}}{2}$, which is implied by $q_{n}<\frac{p_{n}}{2}$.

We now show that $u(x)=\lim _{N \rightarrow \infty} u^{N}(x)$. Since $u^{N}(x)$ is increasing, and $u^{N}(x) \leq u(x)$ for all $N$, it is clear that $\lim _{N \rightarrow \infty} u^{N}(x) \leq u(x)$. To prove the reverse inequality, it suffices to show that $\hat{X}_{T}^{N}$ is a maximizing sequence.

Consider a maximizing sequence for $u(x)$, that is a sequence $\left(Y^{k}\right)_{k=1}^{\infty} \subset \mathcal{X}(x)$ such that $\lim _{k \rightarrow \infty} E\left[U\left(Y^{k}\right)\right]=u(x)$. We may well replace $Y^{k}$ with $\left(1-\delta_{k}\right) Y^{k} 1_{\left\{Y^{k}<M_{k}\right\}}+x \delta_{k}$ for some small $\left(\delta_{k}\right)_{k=1}^{\infty}$ and some big $\left(M_{k}\right)_{k=1}^{\infty}$, so that we still have a maximizing sequence (still denoted by $\left.\left(Y^{k}\right)_{k=1}^{\infty}\right)$, and $Y_{k}$ is bounded away from zero.

As $Y^{k}$ is the terminal payoff of some strategy $H^{k}$, we denote by $Y_{n}^{k}$ the payoff of $H^{k}$ at time $n$. Then we have that $U\left(Y_{n}^{k}\right)$ is bounded from below by $U\left(x \delta_{k}\right)$, uniformly in $n$. Therefore, Fatou's Lemma implies that:

$$
\lim _{n \rightarrow \infty} E\left[U\left(Y_{n}^{k}\right)\right] \geq E\left[U\left(Y^{k}\right)\right]
$$

and hence, choosing $n_{k}$ big enough, $\left(Y_{n_{k}}^{k}\right)_{k=1}^{\infty}$ is a maximizing sequence. On the other hand, we trivially have that $E\left[U\left(Y_{n_{k}}^{k}\right)\right] \leq E\left[U\left(\hat{X}_{T}^{k}\right)\right]$, as desired.

We now prove that $u(x)<\infty$ for all $x>0$. By Theorem 3.1 in KS99 (or by the concavity of $u$ ), it suffices to show that $u(x)<\infty$ for some $x>0$, and we show this for $x^{*}$. The utility maximization problem with initial capital $x^{*}$ admits an optimizer (see also Lemma 2.5 ( $i$ ) below), which is given by:

$$
\hat{X}_{T}\left(x^{*}\right)=\sum_{n=1}^{\infty} 1_{A_{n}} I\left(y^{\infty} \frac{q_{n}}{p_{n}}\right)+1_{B} I\left(y^{\infty} r_{\infty}\right)
$$

By assumption (e) above, $I\left(y^{\infty} \frac{q_{n}}{p_{n}}\right) \in \Xi$ for all $n$. Denoting by $M=U\left(I\left(y^{\infty} r_{\infty}\right)\right)$, we have:

$$
\begin{aligned}
E\left[U\left(\hat{X}_{T}\left(x^{*}\right)\right)\right]<M+2 E\left[\hat{X}_{T}\left(x^{*}\right) U^{\prime}\left(\hat{X}_{T}\left(x^{*}\right)\right) 1_{\Omega \backslash\left(B \cup A_{1}\right)}\right] & = \\
& =M+2 y^{\infty} \sum_{n=2}^{N} I\left(y^{\infty} \frac{q_{n}}{p_{n}}\right) q_{n}=M+2 y^{\infty} x^{*}
\end{aligned}
$$

which shows that $u\left(x^{*}\right)<\infty$.

To show that the optimal strategy $\hat{H}_{n}^{N}$ is nonnegative, we argue by backward induction. The basic idea is very simple: all the investments $1_{A_{n}}-q_{n}$ have a positive expected return, therefore we expect that a utility maximizer will hold them in positive quantities.

Denote by $B_{n}=\Omega \backslash\left(\cup_{i=1}^{n} A_{i}\right)$, the event where $S$ has not jumped during the first $n$ periods. Note that it suffices to prove that $\hat{H}_{n}^{N} 1_{B_{n-1}} \geq 0$, because if the only possible jump 
has already occurred, any further investment is irrelevant, and we may choose $\hat{H}_{n}^{N}=0$ on $\Omega \backslash B_{n-1}$. Observe first that:

$$
E\left[S_{n}-S_{n-1} \mid \mathcal{F}_{n-1}\right]=\alpha_{n}\left(p_{n}-q_{n}\right) \geq \alpha_{n} \frac{p_{n}}{2}>0
$$

and denote by $u_{k}^{N}(x)$ the conditional indirect utility after $k$ time steps, defined as:

$$
u_{k}^{N}(x)=\sup \left\{E\left[U\left(x+\sum_{n=k+1}^{N} \hat{H}_{n}^{N}\left(S_{n}-S_{n-1}\right)\right)\right]: H \text { admissible }\right\}
$$

and note that we trivially have $u_{N}^{N}=U$.

Consider first $n=N-1$. By (18), $S_{N}-S_{N-1}$ has a positive expected return, and the continuity of $U^{\prime}$ implies that the optimal holding $\hat{H}_{N-1}^{N}$ is strictly positive. In particular, this implies that, on the event $B_{N-1}$ :

$$
\left(u_{N-1}^{N}\right)^{\prime}\left(\hat{X}_{N-1}^{N}(x)\right)>U^{\prime}\left(\hat{X}_{N-1}^{N}(x)\right) \text { a.s. }
$$

For $n<N-1$, assume that, for all $k>n, \hat{H}_{k}^{N} \geq 0$ and that

$$
\left(u_{k}^{N}\right)^{\prime}\left(\hat{X}_{k}^{N}(x)\right)>\left(u_{k+1}^{N}\right)^{\prime}\left(\hat{X}_{k}^{N}(x)\right) \text { a.s. on } B_{k}
$$

Again, since $S_{n+1}-S_{n}$ has positive conditional expectation (by $(18)$ ), and $\left(u_{k+1}^{N}\right)^{\prime}$ is continuous (by Theorem 3.1 in [KS99]), it follows that $\hat{H}_{n}^{N}$ must be strictly positive to achieve optimality.

In the next Lemma we describe the properties of the above model:

Lemma 2.5. The model constructed in Lemma 2.4 has the following properties:

i) For all $x \geq x^{*}, \hat{X}_{T}^{N}(x)$ converges to $\hat{X}_{T}\left(x^{*}\right)$ a.s.

ii) $u(x)$ is a straight line with slope $u^{\prime}\left(x^{*}\right)$ for $x>x^{*}$.

iii) Problem $\left(\mathrm{P}\right.$ admits a solution if and only if $x \leq x^{*}$.

Proof.

i) Denoting by $\hat{X}^{N}(x)=\left(\xi_{1}^{N}, \ldots, \xi_{N}^{N}, \mu^{N}\right)$, from 1213 we obtain that, for $x \geq x^{*}$ :

$$
\begin{aligned}
& \lim _{N \rightarrow \infty} \xi_{n}^{N}=I\left(y^{N} \frac{q_{n}}{p_{n}}\right)=I\left(y^{\infty} \frac{q_{n}}{p_{n}}\right) \\
& \lim _{N \rightarrow \infty} \mu^{N}=I\left(y^{N} r_{N}\right)=I\left(y^{\infty} r_{\infty}\right)
\end{aligned}
$$


therefore it suffices to show that this limit coincides with $\hat{X}\left(x^{*}\right)$. In fact, from (11), we obtain that:

$$
\sum_{n=1}^{N-1} I\left(y^{N} \frac{q_{n}}{p_{n}}\right) q_{n}+I\left(y^{N} r_{N}\right)\left(1-\sum_{n=1}^{N} q_{n}\right)=x_{N}
$$

and taking the limit as $N \rightarrow \infty$, we obtain:

$$
\sum_{n=1}^{\infty} I\left(y^{\infty} \frac{q_{n}}{p_{n}}\right) q_{n}+I\left(y^{\infty} r_{\infty}\right)\left(1-\sum_{n=1}^{\infty} q_{n}\right)=x^{*}
$$

which concludes the proof.

Note that for $x<x^{*}$, equations $\left.\begin{array}{ll|l|l|l|l}11 & 12 & 13\end{array}\right)$ still admit a solution, but $y^{N}$ converges to a value strictly greater than $y^{\infty}$.

ii) By Lemma 2.4 we have that $u^{N}(x) \rightarrow u(x)$ for all $x>0$, and by standard results on convex functions (see [Roc97]), this implies that also $\left(u^{N}\right)^{\prime}(x)$ converges to $u^{\prime}(x)$, for all $x>0$. Also, by Theorem 2.0 in [KS99] we have that $\left(u^{N}\right)^{\prime}(x)=y^{N}$.

¿From $i$ ) it follows that, for $x \geq x^{*}$ :

$$
u^{\prime}\left(x^{*}\right)=y^{\infty}=\lim _{N \rightarrow \infty} y^{N}=\lim _{N \rightarrow \infty}\left(u^{N}\right)^{\prime}(x)=u^{\prime}(x)
$$

which proves the claim.

iii) As noted in $i$ ), an optimizer exists for $x=x^{*}$. Then an optimizer exists also for $x<x^{*}$ by Theorem 2.0 in [KS99]. Since an optimizer exists for $x=x^{*}$, then it cannot exist for $x>x^{*}$ by $i i$ ), and by the strict concavity of $U$ (see Scholium 5.1 in [KS99]).

\section{Incorporating Transaction Costs}

In this section we derive, from the example constructed above, a market model with transaction costs where essentially the same phenomenon occurs, as in Lemma 2.4 and 2.5 above.

Let $X$ be a strictly positive, adapted process, and $H$ a strategy, that is a predictable, $X$-integrable process. We define the liquidation value at time $t$ of the strategy $H$ with proportional transaction costs $k$ as $V_{0}^{k}(H, X)=0$ for $t=0$ and, for $t \geq 1$, as:

$$
V_{t}^{k}(H, X)=\sum_{n=0}^{t-1} H_{n}\left(X_{n+1}-X_{n}\right)-k\left(\left|H_{0}\right| X_{0}+\sum_{n=1}^{t-1}\left|H_{n}-H_{n-1}\right| X_{n}+\left|H_{t-1}\right| X_{t}\right)
$$


so that $V_{t}^{0}(H, X)=V_{t}(H)$ with the notation introduced in $(\mathrm{P})$ above.

We now proceed with the proof of Proposition 1.2, which will be broken into three Lemmata. In the first one, from a frictionless model with asset process $S_{n}$, we construct a process $X_{n}$ for which any positive strategy on $S$ (without transaction costs) delivers the same payoff as another strategy on $X$ (with transaction costs $k$ ).

Lemma 3.1. Let $\left(S_{n}\right)_{n=0}^{\infty}$ be an adapted process, and let $\left(X_{n}\right)_{n=0}^{\infty}$ be defined by:

$$
\left\{\begin{array}{l}
X_{0}=x_{0} \\
X_{n+1}=\frac{S_{n+1}-S_{n}}{-\left((-1)^{n}+k\right)}+\frac{1-k(-1)^{n}}{1+k(-1)^{n}} X_{n}
\end{array}\right.
$$

Suppose $X_{n} \geq 0$ a.s. for all $n$. If $(\tilde{H})_{n=0}^{\infty}$ is a nonnegative predictable process, and $\left(H_{n}\right)_{n=0}^{\infty}$ is defined by $H_{n}=(-1)^{n+1} \tilde{H}_{n}$, then

$$
V_{t}^{k}(H, X)=V_{t}^{0}(\tilde{H}, S)
$$

Proof. From (20) it follows that:

$$
S_{n+1}-S_{n}=-(-1)^{n}\left(X_{n+1}-X_{n}\right)-k\left(X_{n+1}+X_{n}\right)
$$

and hence:

$$
V_{t}^{0}(\tilde{H}, S)=\sum_{n=0}^{t-1} \tilde{H}_{n}\left(S_{n+1}-S_{n}\right)=\sum_{n=0}^{t-1} H_{n}\left(X_{n+1}-X_{n}\right)-k \sum_{n=0}^{t-1} \tilde{H}_{n}\left(X_{n+1}+X_{n}\right)
$$

Since $H$ has alternating signs, and $X$ is strictly positive, we have that:

$$
\left|H_{0}\right| X_{0}+\sum_{n=1}^{t-1}\left|H_{n}-H_{n-1}\right| X_{n}+\left|H_{t-1}\right| X_{t}=\sum_{n=0}^{t-1} \tilde{H}_{n}\left(X_{n+1}+X_{n}\right)
$$

which proves the claim.

In the next Lemma, we verify that if $S_{n}$ is a simple jump model, then the above construction leads to a strictly positive process $X_{n}$.

Lemma 3.2. Let $S$ be the risky asset of a simple jump model as in Definition 2.2. Then there exists some $X_{0}>0$ such that $X$ obtained from $S$ by (20) is a strictly positive process.

Proof. Iterating (20), it follows that, for all $n$ even, we have

$$
X_{n}=X_{0}+\sum_{i=1}^{n} \frac{S_{i}-S_{i-1}}{(-1)^{i}(1-k)}
$$


hence we have that:

$$
\left|X_{n}-X_{0}\right| \leq \sum_{i=1}^{n}\left|\frac{S_{i}-S_{i-1}}{(-1)^{i}(1-k)}\right| \leq \frac{1}{1-k} \sum_{i=1}^{n} \alpha_{i}
$$

and therefore $X_{n} \geq X_{0}-\frac{1}{1-k} \sum_{i=1}^{n} \alpha_{i}=M$, which is finite by assumption. If $n$ is even, we also have:

$$
\frac{X_{n+1}}{X_{n}}=\frac{S_{n+1}-S_{n}}{-(1+k) X_{n}}+\frac{1-k}{1+k} \geq \frac{1-k}{1+k}-\frac{\sup _{n} \alpha_{n}}{(1+k) M}
$$

It follows that, by choosing $X_{0}$ large enough, all $X_{n}$ are positive.

Finally, we check that any strategy on $X$ with transaction costs is dominated by another one in $S$ without transaction costs.

Lemma 3.3. Let $\left(S_{n}\right)_{n=0}^{\infty}$ be an adapted process, and $\left(X_{n}\right)_{n=0}^{\infty}$ be defined as in Lemma 3.1. Suppose $X_{n} \geq 0$ a.s. for all $n$. If $\left(H_{n}\right)_{n=0}^{\infty}$ is any strategy (i.e. a predictable $\mathbb{R}$-valued process), and $\tilde{H}_{n}=(-1)^{n+1} H_{n}$, then we have that:

$$
V_{t}^{k}(H, X) \leq V_{t}^{0}(\tilde{H}, S) \quad \text { a.s. } \quad \text { for } 0 \leq t \leq T
$$

Proof. We prove the claim by induction on $t$. For $t=0$, we trivially have $V_{t}^{k}(H, X)=$ $V_{t}^{0}(\tilde{H}, S)=0$. Suppose that the claim is true for $s<t$. We define the stopping times:

$$
\begin{aligned}
& \tau=\inf \left\{n \geq 0: \tilde{H}_{n}<0\right\} \\
& \sigma=\inf \left\{n>\tau: \tilde{H}_{n} \geq 0\right\}
\end{aligned}
$$

and note that $\sigma \geq 1$. If $\tau \geq t$, then $\tilde{H}_{t}$ is nonnegative, and the thesis follows from Lemma 3.1. For $t>\tau$, adding and subtracting both $k\left|H_{\tau-1}\right| X_{\tau}$ and $k\left|H_{\sigma}\right| X_{\sigma}$, we have:

$$
\begin{aligned}
V_{t}^{k}(H, X) & =V_{\tau}^{k}(H, X)+ \\
& +k\left|H_{\tau-1}\right| X_{\tau}-k \sum_{n=\tau}^{\sigma}\left|H_{n}-H_{n-1}\right| X_{n}+k\left|H_{\sigma}\right| X_{\sigma}+\sum_{n=\tau}^{\sigma-1} H_{n}\left(X_{n+1}-X_{n}\right)+ \\
& +V_{t-\sigma}^{k}\left(H_{-\sigma}, X ._{-\sigma}\right)
\end{aligned}
$$

where $H_{{ }_{-\sigma} \sigma}$ and $X_{._{-\sigma}}$ are the processes defined by $\left(H_{{ }_{-\sigma}}\right)(t)=H_{t-\sigma}$ and $\left(X_{{ }_{-\sigma}}\right)(t)=X_{t-\sigma}$ respectively.

By the inductive hypothesis, (25) and (27) are respectively less than $V_{\tau}^{0}(\tilde{H}, S)$ and $V_{t-\sigma}^{0}\left(\tilde{H}_{-\sigma \sigma}, S_{-\sigma}\right)$. The second term in 26 can be estimated from above as:

$$
-k \sum_{n=\tau}^{\sigma}\left|H_{n}-H_{n-1}\right| X_{n} \leq-k\left(\left|H_{\tau}-H_{\tau-1}\right| X_{\tau}+\left|H_{\sigma}-H_{\sigma-1}\right| X_{\sigma}\right)
$$


For $\tau \leq n \leq \sigma-1$, we have that $H_{n}=-\left|H_{n}\right|(-1)^{n+1}$. Also, recall that (21) implies that:

$$
X_{n+1}-X_{n}=(-1)^{n+1}\left(S_{n+1}-S_{n}+k\left(X_{n+1}-X_{n}\right)\right)
$$

and hence the last term in $(26)$ can be written as:

$$
\begin{aligned}
\sum_{n=\tau}^{\sigma-1} H_{n}\left(X_{n+1}-X_{n}\right) & =-\sum_{n=\tau}^{\sigma-1}\left|H_{n}\right|\left(S_{n+1}-S_{n}\right)-k \sum_{n=\tau}^{\sigma-1}\left|H_{n}\right|\left(X_{n+1}+X_{n}\right) \leq \\
& \leq-\sum_{n=\tau}^{\sigma-1}\left|H_{n}\right|\left(S_{n+1}-S_{n}\right)-k\left|H_{\tau}\right| X_{\tau}-k\left|H_{\sigma-1}\right| X_{\sigma}
\end{aligned}
$$

Note that the last inequality holds as an equality in the case $\tau=\sigma-1$. Substituting (30) and $(28)$ in (26), we finally obtain:

$$
\begin{aligned}
\sqrt[26]{26} \leq-\sum_{n=\tau}^{\sigma-1}\left|H_{n}\right|( & \left.S_{n+1}-S_{n}\right)+k X_{\tau}\left(\left|H_{\tau-1}\right|-\left|H_{\tau}\right|-\left|H_{\tau}-H_{\tau-1}\right|\right)+ \\
& +k X_{\sigma}\left(\left|H_{\sigma}\right|-\left|H_{\sigma-1}\right|-\left|H_{\sigma}-H_{\sigma-1}\right|\right) \leq-\sum_{n=\tau}^{\sigma-1}\left|H_{n}\right|\left(S_{n+1}-S_{n}\right)
\end{aligned}
$$

where the last inequality follows by the triangular inequalities $\left|H_{\tau-1}\right| \leq\left|H_{\tau}\right|+\left|H_{\tau}-H_{\tau-1}\right|$ and $\left|H_{\sigma}\right| \leq\left|H_{\sigma-1}\right|+\left|H_{\sigma}-H_{\sigma-1}\right|$. Summing up, we have:

$$
\begin{aligned}
V_{t}^{k}(H, X) & \leq V_{\tau}^{0}(\tilde{H}, S)+V_{t-\sigma}^{0}\left(\tilde{H}_{-\sigma}, S \cdot_{-\sigma}\right)-\sum_{n=\tau}^{\sigma-1}\left|H_{n}\right|\left(S_{n+1}-S_{n}\right)= \\
& =V_{\tau}^{0}(\tilde{H}, S)+V_{t-\sigma}^{0}\left(\tilde{H}_{._{-\sigma}}, S ._{-\sigma}\right)+\sum_{n=\tau}^{\sigma-1} \tilde{H}_{n}\left(S_{n+1}-S_{n}\right)= \\
& =V_{t}^{0}(\tilde{H}, S)
\end{aligned}
$$

which completes the proof.

We can now resume the above results as follows:

Proof of Proposition 1.2. Let $S$ be the simple jump model (as in Definition 2.2) constructed as in Lemma 2.4. The properties of this model are resumed in Proposition 2.1.

If we define $X$ as in Lemma 3.1, we obtain a positive process by Lemma 3.2. Define the value functions of the two utility maximization problems as:

$$
\begin{gathered}
u_{S}(x)=\sup \left\{E\left[U\left(x+V_{T}^{0}(H, S)\right)\right]: H \text { admissible }\right\} \\
u_{X}(x)=\sup \left\{E\left[U\left(x+V_{T}^{k}(H, X)\right)\right]: H \text { admissible }\right\}
\end{gathered}
$$


By Lemma 2.4, there exists a sequence of maximizing strategies for $u_{S}$ with positive holdings. Then, by Lemma 3.1 it follows that $u_{S}(x) \leq u_{X}(x)$, and the reverse inequality follows from Lemma 3.3 . This proves $i$ ) and $i i$ ). To prove $i i i)$, note that $u_{X}$ has a maximizer if and only if $u_{S}$ has one.

\section{A Appendix}

Lemma A.1. Let $U$ be a utility function satisfying Assumption 1.1 and (2). For all $\varepsilon>0$, there is $x>\varepsilon^{-1}$ such that:

i) $x U^{\prime}(x) \geq \varepsilon^{-1}$

ii) $\frac{z U^{\prime}(z)}{U(z)} \geq 1-\varepsilon$ for all $z \in\left[\varepsilon x, \frac{x}{\varepsilon}\right]$

iii) $U^{\prime}(\varepsilon x) \leq(1+\varepsilon) U^{\prime}(x)$

Notice that $i$ i) is an immediate consequence of (2), while $i i i$ ) follows from Lemma 6.5 in [KS99]. The difficulty of the above Lemma consists in finding some $x$ which satisfies simultaneously $i$ ) and $i$ ii).

The proof requires two auxiliary Lemmata.

Lemma A.2. Let $U$ be a utility function satisfying Assumption 1.1, and $y<x$ such that:

$$
\frac{y U^{\prime}(y)}{U(y)} \leq \alpha \quad \frac{x U^{\prime}(x)}{U(x)} \geq \beta
$$

Then we have that:

$$
x \geq y\left(\frac{\beta-\alpha}{(1-\beta) \alpha}+1\right)
$$

Proof. Define $b(x)=U(x)-x U^{\prime}(x)$. Note that $b(x)$ can be characterized as:

$$
b(x)=\inf \left\{m: U^{\prime}(x) z+m \geq U(z) \text { for all } z>0\right\}
$$

and therefore $b(x)$ is an increasing function of $x$ (when $U$ is twice differentiable, this is immediately seen by differentiating the definition of $b(x))$. This fact, combined with the assumptions on $x$ and $y$, implies that:

$$
(1-\alpha) U(y) \leq b(y) \leq b(x) \leq(1-\beta) U(x)
$$

and by the concavity of $U$ :

$$
U(x) \leq U(y)+U^{\prime}(y)(x-y)
$$


Putting together (33) and (34), we have:

$$
x-y \geq \frac{\beta-\alpha}{1-\beta} \frac{U(y)}{U^{\prime}(y)}
$$

and hence:

$$
x \geq y\left(\frac{\beta-\alpha}{1-\beta} \frac{U(y)}{y U^{\prime}(y)}+1\right) \geq y\left(\frac{\beta-\alpha}{(1-\beta) \alpha}+1\right)
$$

Lemma A.3. Let $U$ be a utility function satisfying Assumption 1.1, and $U(\infty)>0$. Let $0<y<x$ and $0<\alpha<\beta$.

i) If $\frac{x U^{\prime}(x)}{U(x)} \geq \beta$ and $\frac{z U^{\prime}(z)}{U(z)} \geq \alpha$ for all $z \in[y, x]$ then $U^{\prime}(x)>\frac{\beta}{\alpha}\left(\frac{x}{y}\right)^{\alpha-1} U^{\prime}(y)$

ii) For $y$ large enough, if $\frac{y U^{\prime}(y)}{U(y)} \geq \beta$ and $\frac{x U^{\prime}(x)}{U(x)} \leq \alpha$ then $U^{\prime}(y) \geq \frac{\beta}{\alpha} U^{\prime}(x)$

Proof.

i) By Gronwall's lemma, we have:

$$
U(z) \geq U(y)\left(\frac{z}{y}\right)^{\alpha} \quad \text { for all } z \in[y, x]
$$

It follows that:

$$
\beta \leq \frac{x U^{\prime}(x)}{U(x)} \leq \frac{y U^{\prime}(x)}{U(y)}\left(\frac{y}{x}\right)^{\alpha-1} \leq \alpha \frac{U^{\prime}(x)}{U^{\prime}(y)}\left(\frac{y}{x}\right)^{\alpha-1}
$$

which completes the proof.

ii) Notice that:

$$
\left(\frac{U(x)}{x}\right)^{\prime}=\frac{x U^{\prime}(x)-U(x)}{x^{2}}=-\frac{b(x)}{x^{2}}
$$

Since $U(\infty)>0$ implies that $\lim _{x \rightarrow \infty} b(x)>0$, it follows that $\frac{U(x)}{x}$ is a decreasing positive function on the nonempty set $\{x: b(x) \geq 0, U(x) \geq 0\}$. It follows that:

$$
\frac{U^{\prime}(y)}{U^{\prime}(x)} \geq \frac{\beta}{\alpha} \frac{U(y)}{y} \frac{x}{U(x)} \geq \frac{\beta}{\alpha}
$$

as claimed. 
Proof of Lemma A.1. First note that $A E_{+\infty}(U)>0$ implies that $U$ is unbounded (Lemma 6.1 in [KS99]). Hence, we only need to find some $x$ big enough which satisfies $i i$ ) and $i i i)$, as $i$ ) will follow automatically.

If there exists some $x_{0}$ such that $\frac{x U^{\prime}(x)}{U(x)} \geq 1-\varepsilon$ for all $x \geq x_{0}$, the result follows by Lemma 6.5 in [KS99]. In fact, from the mentioned lemma we obtain that, for all $\alpha<1$ and $\beta>1$ there exists $y>\varepsilon^{-1}$ such that:

$$
\alpha U^{\prime}(y)<U^{\prime}(\beta y)
$$

and $i i i$ ) follows by setting $\beta=\frac{1}{\varepsilon}, x=\frac{y}{\varepsilon}$ and $\alpha=\frac{1}{1+\varepsilon}$. Such an $x$ will then satisfy $\left.i i\right)$ and i) above.

Otherwise, if $\liminf \operatorname{in}_{x \rightarrow \infty} \frac{x U^{\prime}(x)}{U(x)}<1$, then there exist $y, \hat{x}$, with $y<\hat{x}$ such that:

$$
\frac{\hat{x} U^{\prime}(\hat{x})}{U(\hat{x})} \geq 1-\varepsilon^{3}, \quad \frac{y U^{\prime}(y)}{U(y)}=1-\varepsilon \quad \text { and } \quad \frac{z U^{\prime}(z)}{U(z)} \geq 1-\varepsilon \quad \text { for all } z \in[y, \hat{x}]
$$

In fact, we may just choose some $y<\hat{x}$ with the first two properties, and then replace $y$ with $\sup \left\{w<\hat{x}: \frac{w U^{\prime}(w)}{U(w)} \leq 1-\varepsilon\right\}$.

Denote now $x=\inf \left\{w>y: \frac{w U^{\prime}(w)}{U(w)}=1-\varepsilon^{2}\right\}$. Notice that, by Lemma A.2, we have:

$$
\hat{x} \geq\left(1+\frac{1}{\varepsilon(1+\varepsilon)}\right) x \geq \frac{x}{\varepsilon} \quad \text { and } \quad \varepsilon x \geq y(1+\varepsilon)>y
$$

Applying Lemma A.3 (i) to $y=\varepsilon x$, we obtain that:

$$
U^{\prime}(x) \geq(1+\varepsilon) \varepsilon^{\varepsilon} U^{\prime}(\varepsilon x)>(1+\varepsilon)(1+\varepsilon \log \varepsilon) U^{\prime}(\varepsilon x)
$$

and $i$ ii) follows for $\varepsilon$ small enough.

\section{References}

[Gua02] Paolo Guasoni, Optimal investment with transaction costs and without semimartingales, Ann. Appl. Probab. 12 (2002), no. 4, 1227-1246. MR 2003h:91063

[KS99] D. Kramkov and W. Schachermayer, The asymptotic elasticity of utility functions and optimal investment in incomplete markets, Ann. Appl. Probab. 9 (1999), no. 3, 904-950. MR 2001d:91099

[Roc97] R. Tyrrell Rockafellar, Convex analysis, Princeton Landmarks in Mathematics, Princeton University Press, Princeton, NJ, 1997, Reprint of the 1970 original, Princeton Paperbacks. MR 97m:49001 
[Sch02] Walter Schachermayer, Optimal investment in incomplete financial markets, Mathematical finance-Bachelier Congress, 2000 (Paris), Springer Finance, Springer, Berlin, 2002, pp. 427-462. MR 1960575 